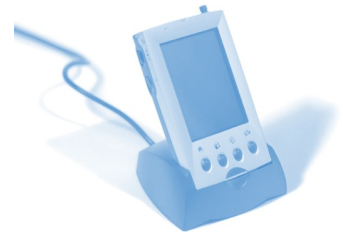

Mike Batham, Kate Brashaw, Rob Janes* and Ruth Williams Department of Chemistry and Analytical Sciences and Faculty of Science SWIM group

The Open University

Walton Hall

Milton Keynes

MK7 6AA

*r.janes@open.ac.uk

\section{Development of a web-based teaching resource for analytical science practitioners}

\begin{abstract}
This paper describes the production of multimedia teaching material aimed at working analysts in the water industry who are studying the Open University's Foundation Degree in Analytical Sciences. In collaboration with staff at Scottish Water, Edinburgh laboratories, audio and video materials were produced which demonstrate a number of basic laboratory techniques routinely carried out in chemistry and microbiology laboratories. Teaching laboratory techniques (and associated safety procedures) is by its very nature a visual process, and requires the cultivation of a skill base and good practice that can only be gained by repetition. However an initial 'show and tell' stage is required. This is extended to coverage of aspects of laboratory management, and how analysts work in teams to produce analytical data for the customer.
\end{abstract}

\section{Background}

The Department of Chemistry and Analytical Sciences at the Open University is currently working closely with industrial partners to produce a Foundation Degree in Analytical Sciences. Our 30 credit, level 1 work-based learning module (taught entirely on-line) is designed to complement the student's existing practice skills, and develop their understanding of the science underpinning the procedures and equipment used in their professional practice in chemistry and microbiology laboratories.

One outcome of meetings with an advisory group comprising managers and training officers from the analytical sector (specifically from laboratories dealing with water analysis) has been the identification of a need for high quality multimedia material to support the teaching of laboratory techniques in an applied context. This is not to say that excellent teaching material is not available; indeed in this module we include embedded links to Chemical Video Consortium videos ${ }^{1}$, and indeed on researching this project, resources such as video and pre-lab material from the Bristol CETL ${ }^{2}$, existing OU produced material and the plethora of clips available on YouTube were reviewed. However our feeling was that for the purposes of a work-based learning module such as this, the production of bespoke material, tailored to our target sector was preferable. A further outcome of these meetings was a strong steer in the direction of the most appropriate techniques to cover; in some cases apparatus not generally found in a conventional University teaching laboratory (e.g. a Turbovap for solvent removal) were included. From a practical standpoint, an invitation to use the laboratories of Scottish Water, Edinburgh as a production base was gratefully accepted.

Taking a step back though, the phrase 'in an applied context' in the previous paragraph might appear somewhat trite. However, the ethos of this project was to extend beyond the science and create multimedia material which provided a holistic view of the analytical process, firstly by showing 'real analyses' going on in a 'real laboratory' incorporating some of the generic and transferable skills involved. To this end, two video podcasts were produced which followed two analytical operations from receipt of samples, logging on to LIMS (Laboratory Information Management System), through to completion, i.e. the production of fit for purpose data for the customer. Both integrate aspects of the science, presented in an accessible manner, with details of how these operations are managed, and the important function of team working in the laboratory. To add further value we introduced students to the analysts carrying out the work, giving them the opportunity to talk about their work, in particular how they relate to others in their team, and the necessity for good communication skills. As part of the work based learning module we ask students to reflect on how their own proficiency in these areas can be improved. 
In a broader sense, production of this resource forced us to think about what our work-based learning students, really take from a module such as this to develop their own appreciation of laboratory methods. For example, does a student routinely using a balance several times a day, really need us to tell her/ him how it should be done? The answer is probably no, but this is something that we are clear about from the start. We appreciate that students will most likely skate through some of the content quite quickly, but we make it clear that although they may be routinely using some of the techniques covered, they may not be using them all and so the material serves to enrich the student's knowledge. In addition, we ask students to think about how particular operations are carried out in their working environment, whether it be the etiquette involved in using a communal balance or differences between 'our way' of carrying out a particular operation with what takes place in the student's laboratory.

A summary of the content of the resources produced in the laboratories of Scottish Water, is given in the following section.

\section{... production of this resource forced us to think about what our work-based learning students, really take from a module such as this to develop their own appreciation of laboratory methods.}

\footnotetext{
Deliverables

i) Video podcast:

Monitoring of Cryptosporidium in Water: this covers a routine procedure for the detection of the oocysts of this pathogenic organism. The process begins with a sample of filtered water from a customer from a pumping station, this is filtered and oocysts are trapped. The filter is then washed and the solution is concentrated. The technique of immunomagnetic separation is then demonstrated, in which the oocysts are separated from any extraneous particles by using nanosized iron beads coated with antibodies which specifically lock on to Cryptosporidium, which are then removed magnetically. Finally a staining procedure is carried out and oocysts (if present) are identified under the fluorescent light microscope. Along the way, students are introduced to such processes as centrifugation, slide preparation and incubation.
}

\section{ii) Video podcast:}

Analysis of Organics in Water: this covers the theory of solvent extraction, and how separation may be effected using first a separating funnel, and then mechanical shakers to enable the laboratory to cope with a high throughput of samples. This is followed by a demonstration of evaporation of solvent under reduced pressure to increase the concentration of analyte in the sample, which is subjected to analysis by gas chromatography, and the presence of organic acids, a common indicator of sewage pollution, was confirmed.

\section{iii) Interviews with laboratory personnel:}

Audio files were also produced consisting of interviews with two scientists and one senior manager at Scottish Water. In response to scripted questions interviewees were asked to consider a number of issues: their day-to-day role; how good team working and communication ensures a constant flow of work and delivery of high quality analytical data; how team work is encouraged and supported; and the central role in this of an effective appraisal and review system.

iv) short video clips and stills sequences:

These illustrated how key analytical techniques are carried out by working analysts. These included a closer look at solvent extraction, solid phase extraction, $\mathrm{pH}$ measurement, aseptic technique and aspects of laboratory health and safety.

Finally, a few words about the practicalities: we found that thorough pre-planning was essential. This included two visits to Scottish Water to agree content with a laboratory manager, followed by producing a filming and recording schedule and preparing a script of questions for audio interviews (interviewees were provided with the questions prior to recording). Following filming, the films/audio recordings were edited and scripts for voice-over commentaries prepared and recorded. A sample script, simply to give an indication of coverage, is included below.

\section{Sample voice-over script: solid phase extraction.}

Solid phase extraction is widely used to purify and concentrate samples prior to analysis. A solid, often but not always, silica based, is packed into a syringe-like cartridge, this is known as the stationary phase.

A common procedure is to chemically bond organic functional groups to the surface of silica, one example being the octadecyl -, or $C_{18}$ group. Non-polar organic substances are attracted by Van de Waals forces at these points. This concentrates them on the column. They are then washed off the solid support by a suitable solvent and out through the bottom of the column - a process known as elution.

An alternative approach is to remove the interfering substances on the column and let the sample of interest run through.

Here the cartridges are incorporated into a manifold. This is connected to a vacuum pump which speeds up the extraction process by drawing the liquid sample through the stationary phase. You may also encounter the stationary phase in the form of flat discs or an array of 96 well plates which are used for tiny volumes of sample. 
What you have been watching so far is an initial step whereby the column is conditioned. The octadecyl centres tend to clump together - the analyst here is passing a solution of formic acid in methanol through the column which opens up the $C_{18}$ groups allowing for more effective capture of the analyte. Next the column is flushed through with water to remove as much methanol as possible - this is important as methanol is going to be used in the elution step later on.

Now we begin the extraction. The sample is siphoned into the column via a plastic tube. You'll also notice that although we're only showing one cartridge here, there is in fact space for others to run simultaneously. Note the flow rate into the column remains steady at about 5 drops per second at this stage. The waste passes through the column into a trough, again note the rapid flow rate.

Once our sample has passed through the column, the tube is removed, a few taps to make sure all the sample has passed through, and now we move on to the elution stage. The glass collection vessel is thoroughly cleaned and a plastic housing containing a collection tube has been inserted. The elution solution is added. You'll notice the flow rate is much slower in this step - this to optimise the recovery of the analyte.

One example of the use of solid phase extraction is drugs testing in sport. An athlete's urine is passed through a $C_{18}$ -silica column that retains steroids and other organic molecules - whereas polar and ionic substances pass straight through. The retained compounds are then eluted using a solvent such as hexane. This solvent would then be evaporated and the residue dissolved in the minimum volume of solvent required for analysis using chromatography.

\section{Conclusions and future work}

Our experiences producing the multimedia materials described above provided a template for a way of working with industry to produce teaching materials, which we wish to carry forward. We plan to make this material accessible to all by inclusion as podcasts on iTunesU, and as content on the Open University dedicated channel on YouTube - OU View. As the Analytical Sciences programme at the Open University grows we plan to build on this work and to produce analogous materials working with other areas of the analytical sector. In a more general sense the production of this material placed us in a situation where we were forced to think about curriculum in a work-based learning context and what material is appropriate to students. In a general sense our focus was on coverage of the underlying chemistry and extending to laboratory techniques a student might not be crucial to this was a strong steer provided by our industrial collaborators.

\section{Acknowledgement}

We are very grateful to the staff of Scottish Water (Edinburgh) who were involved in this project, and in particular to Mr Simon Gillespie for his enthusiastic support.

The project was funded by the Centre for Practice-based Professional Learning at the Open University.

\section{References}

1. Chemical Video Consortium, Practical laboratory Chemistry, University of Southampton.

2. Bristol ChemLabS Dynamic Laboratory Manual, University of Bristol.

\section{Our experiences producing the multimedia materials described above provided a template for a way of working with industry to produce teaching materials, which we wish to carry forward.}

